\title{
Production of Terpenoids by Synthetic Biology Approaches
}

\author{
Caizhe Zhang and Kui Hong* \\ Key Laboratory of Combinatorial Biosynthesis and Drug Discovery, Ministry of Education, School of Pharmaceutical \\ Sciences, Wuhan University, Wuhan, China
}

\section{OPEN ACCESS}

Edited by:

Shihui Yang,

Hubei University, China

Reviewed by:

Jifeng Yuan,

Xiamen University, China

Zhihua Zhou,

Shanghai Institutes for Biological Sciences (CAS), China

Lee Seung-Goo,

Korea Research Institute

of Bioscience and Biotechnology

(KRIBB), South Korea

${ }^{*}$ Correspondence:

Kui Hong

kuihong31@whu.edu.cn

Specialty section:

This article was submitted to Synthetic Biology,

a section of the journal

Frontiers in Bioengineering and Biotechnology

Received: 21 December 2019 Accepted: 30 March 2020 Published: 24 April 2020

Citation:

Zhang C and Hong K (2020) Production of Terpenoids by Synthetic

Biology Approaches.

Front. Bioeng. Biotechnol. 8:347.

doi: 10.3389/fbioe.2020.00347
Terpenoids are a large family of natural products with remarkable diverse biological functions, and have a wide range of applications as pharmaceuticals, flavors, pigments, and biofuels. Synthetic biology is presenting possibilities for sustainable and efficient production of high value-added terpenoids in engineered microbial cell factories, using Escherichia coli and Saccharomyces cerevisiae which are identified as well-known industrial workhorses. They also provide a promising alternative to produce non-native terpenes on account of available genetic tools in metabolic engineering and genome editing. In this review, we summarize the recent development in terpenoids production by synthetic biology approaches.

Keywords: terpenoids, synthetic biology, metabolic engineering, Escherichia coli, Saccharomyces cerevisiae

\section{INTRODUCTION}

Terpenoids, also known as terpenes or isoprenoids, are a large family of natural products. More than 80,000 different terpenoids have been found in almost all life forms. Structural diversity of terpenoids makes them a wide range of applications as pharmaceutical, biofuels, and flavors. The skeletons of terpenoids are derived by condensation of multiple units of isopentenyl diphosphate (IPP) and its isomer dimethylallyl diphosphate (DMAPP), which are naturally generated by either mevalonate (MVA) pathway in eukaryotes and methylerythritol-phosphate (MEP) pathway in prokaryotes and plant plastids. In addition to these natural routes, synthetic routes for non-natural precursors have also been reported (Kang et al., 2016; Chatzivasileiou et al., 2019; Clomburg et al., 2019). Core structures of terpenes are then post-modified by cytochromes P450s (P450s) that play a vital role in endowing various bioactivities to terpenoids.

Production of terpenoids from natural resources may encounter technical challenges. For instance, ginsenoside Rh2, a potent candidate for cancer therapy, is a triterpenoid saponin derived from Panax species (Wong et al., 2015). Its content in dried Panax ginseng roots is less than $0.01 \%$ (Wang et al., 2019). Using the synthetic biology approach in engineered yeast, the yield of ginsenoside $\mathrm{Rh} 2$ has reached $2.25 \mathrm{~g} / \mathrm{L}$ in fed-batch fermentation (Wang et al., 2019). This result provides an excellent case for improving cell factories to produce plant rare natural products. The rapid advances in synthetic biology suggest an alternative sustainable approach to achieve the industrial scale of terpenoids production (Bian et al., 2017; Clomburg et al., 2017; Wang et al., 2018). However, several significant challenges remain in microbial biosynthesis as a general approach for the supply of valuable terpenoids, including (i) the biological parts for genetic circuits construction have not been sufficiently characterized; (ii) the post-modifications of terpenoids remains inefficient; and (iii) the toxic accumulation of intermediate products and insufficient supply of precursors. Therefore, a platform that can provide available genetic tools and a comprehensive understanding of its metabolism is urgently needed. In this purpose, Escherichia 
coli and Saccharomyces cerevisiae have been used as ideal platform hosts for various creative explorations (Table 1). In this review, we focus on recently developed strategies specific to address challenges that in the pathway efficiencies optimization, gene circuits construction and regulation, pathway programing, subcellular engineering and co-culture strategy of terpenoids biosynthesis using synthetic biology approaches.

\section{GENETIC CIRCUITS AND DYNAMIC CONTROL}

As the basic genetic elements of biosynthetic pathways, biological parts (e.g., promoter, terminator, ribosome-binding site (RBS), regulatory protein, etc.) should be well-characterized and optimized for synthetic biology. Constitutive or inducible promoters with different strengths are always the major theme in synthetic biology. Their efficiency are also affected by the combination of terminators and RBS.

Comparing to constitutive promoters, inducible promoters possess a strong capacity to start gene expression only under specific culture conditions. For example, the glucosesensing promoter $H X T 1\left(\mathrm{P}_{H X T 1}\right)$ is strong at high glucose concentrations and weak at low glucose concentrations. Using glucose-responsive promoters also avoids the need for expensive repressors or inducers (Scalcinati et al., 2012; Xie et al., 2015; Zhao et al., 2017; Cheng et al., 2019). By using $\mathrm{P}_{\text {HXT1 }}$, the competing gene farnesyl diphosphate synthase (ERG20) for the consumption of precursors IPP and DMAPP was inhibited, and the carbon flux was reallocated from the growth pathway to the limonene synthetic pathway, and the limonene titer reached $917.7 \mathrm{mg} / \mathrm{L}$ in fed-batch fermentation (Cheng et al., 2019). When each of MVA pathway enzymes was transcribed from high-expression galactose-regulated promoters $\left(P_{G A L 1}\right.$ or $\left.P_{G A L 10}\right)$, an amorpha-4,11-diene yield of more than $40 \mathrm{~g} / \mathrm{L}$ was resulted (Westfall et al., 2012). FPP (farnesyl diphosphate) is the intermediate of MVA pathway, but exhibits toxicity when it accumulates in E. coli (Martin et al., 2003). Wholegenome transcript arrays identified an FPP-responsive promoter answering to the accumulation of FPP (Dahl et al., 2013). Using IPP/FPP-responsive promoter in E. coli, Shen et al. (2016) coordinated the expression of all genes of the MVA pathway from $S$. cerevisiae using the tunable intergenic regions to increase the availability of FPP. The dynamically regulated MVA pathway prevented the toxic accumulation of IPP/FPP, and the titer of zeaxanthin reached $722.46 \mathrm{mg} / \mathrm{L}$ in fed-batch fermentation (Supplementary Figure S1A). $\mathrm{P}_{E R G 1}$ represents an ergosterolsensitive promoter, was shown to restrict squalene synthase (ERG9) expression levels efficiently (Yuan and Ching, 2015). Callari et al. (2018) replaced the promoter regions of ERG20 and ERG9 with $\mathrm{P}_{H X T 1}$ and $\mathrm{P}_{E R G 1}$ to redirect the flux from FPP and sterols, generated a titer of $108.5 \mathrm{mg} / \mathrm{L}$ of casbene.

Clustered regularly interspaced short palindromic repeats interference (CRISPRi) uses a catalytically-inactive Cas9 protein (dCas9) and a single guide RNA (gRNA) to repress the expression of targeted genes by blocking transcription (Qi et al., 2013). Kim et al. (2016) established a dynamic regulation CRISPRi system to coordinate the metabolic flux between cell growth and IPP/DMAPP accumulation. An L-rhamnose-inducible promoter was used to control the expression of dCas9. During the production phase, L-rhamnose was removed to restore gene expression, and the production of lycopene and $\alpha$-bisabolol increased. Lian et al. (2017) developed a CRISPR-AID system using three orthogonal CRISPR proteins combines. When HMG1 was overexpressed, down-regulation of ERG9 and deletion of ROX 1 could significantly increase the production of $\beta$-carotene in $S$. cerevisiae. These genes were chosen as the targets for CRISPRa (transcriptional activation), CRISPRi, and CRISPRd (gene deletion), respectively (Supplementary Figure S1B).

The modular pathway engineering group multiple genes into modules to reduce regulatory complexities and help to unlock the potential of the multi-gene pathway for the production of terpenoid products (Ajikumar et al., 2010; Supplementary Figure S1C). Keasling's group tuned the expression of multiple genes within operons by generating libraries of tunable intergenic regions and balancing the expression of MVA pathway, which resulted in a 7 -fold increase in mevalonate production (Pfleger et al., 2006). Zhang et al. (2018b) reported a multidimensional heuristic process for astaxanthin production. Astaxanthin biosynthesis pathway was grouped into four modules, that each module controlled by different promoter of pre-determined strength, and get a yield of $320 \mathrm{mg} / \mathrm{L}$ in E. coli. Through screening of combinations of promoters and terminators, valencene synthase expression cassette was optimized to reach a titer of $539.3 \mathrm{mg} / \mathrm{L}$ (Chen et al., 2019). When Shukal et al. (2019) introduced viridiflorol synthase (VS) from Agrocybe aegerita to E. coli, three T7 promoter variants were characterized for different pathway expression, and RBS libraries that covered a broad range of translational initiation rates were optimized. The yield of viridiflorol was increased to $25.7 \mathrm{~g} / \mathrm{L}$ in fedbatch fermentation.

\section{PATHWAY ENZYME DESIGN}

Directed evolution and rational protein design have been used to engineer enzymes in heterologous pathways (Eriksen et al., 2014; Niu et al., 2018; Hong et al., 2019; Supplementary Figure S1E). Monoterpenes are synthesized from geranyl diphosphate (GPP), which is also the precursor for the biosynthesis of FPP. Therefore, preventing the consumption of GPP by restricting FPP formation is profitable to produce monoterpenes. Mendez-Perez et al. (2017) introduced a mutation (Ser81 to Phe) in native FPP synthase of $E$. coli, resulting in an enzyme that preferentially synthesizes GPP instead of FPP, and the engineered strains yielded $653 \mathrm{mg} / \mathrm{L}$ of 1,8-cineole and $505 \mathrm{mg} / \mathrm{L}$ of linalool, which are 30- and 5-fold improvement, respectively. Jiang et al. (2017) demonstrated that two essential amino acid residues Y436 and D501 located in active pocket of the key enzyme geraniol synthase are critical for the key step of dephosphorylation. By overexpression of truncated 3-hydroxy-3-methylglutarylcoenzyme reductase (tHMGR) and isopentenyl diphosphate isomerase (IDI), the highest titer of $1.68 \mathrm{~g} / \mathrm{L}$ geraniol was achieved in fed-batch fermentation in S. cerevisiae. 
TABLE 1 | Strategies for production of various terpenoids in S. cerevisiae and E. coli

\begin{tabular}{|c|c|c|c|c|}
\hline Product & Strategy and features & Culture conditions & Titer or Improvement & References \\
\hline \multicolumn{5}{|l|}{ S. cerevisiae } \\
\hline 8-hydroxygeraniol & $\begin{array}{l}\text { Mitochondrial compartmentalization by targeting the geraniol biosynthetic pathway to the } \\
\text { mitochondria }\end{array}$ & Fed-batch fermentation & $227 \mathrm{mg} / \mathrm{L}$ & Yee et al., 2019 \\
\hline Geraniol & Protein structure analysis, site-directed mutation, overexpression of tHMGR and IDI & Fed-batch fermentation & $1.68 \mathrm{~g} / \mathrm{L}$ & Jiang et al., 2017 \\
\hline \multirow[t]{2}{*}{ Limonene } & Regulation of ERG20 by $\mathrm{P}_{\mathrm{HXT} 1}$ promoter (glucose-sensitive) & Fed-batch fermentation & $917.7 \mathrm{mg} / \mathrm{L}$ 6-fold & Cheng et al., 2019 \\
\hline & $\mathrm{N}$-degron-mediated destabilization of ERG20 & Batch fermentation & $76 \mathrm{mg} / \mathrm{L}$ & Peng et al., 2018 \\
\hline \multirow[t]{2}{*}{ Amorpha-4,11-diene } & $\begin{array}{l}\text { Optimization of [NADPH]/[NADP }+ \text { ] ratios by introducing mutations into phosphofructokinase (PFK) } \\
\text { along with overexpression of ZWF1 }\end{array}$ & Shake flasks & $497 \mathrm{mg} / \mathrm{L}$ & Kwak et al., 2019 \\
\hline & $\begin{array}{l}\text { Mitochondria compartmentalization by targeting the whole FPP pathway together with } \\
\text { Amorpha-4,11-diene synthase (ADS) into mitochondria }\end{array}$ & Shake flasks & $427 \mathrm{mg} / \mathrm{L}$ & Yuan and Ching, 2016 \\
\hline Zerumbone & Regulation of ERG9 by $\mathrm{P}_{\mathrm{HXT} 1}$ promoter & Fed-batch fermentation & $40 \mathrm{mg} / \mathrm{L}$ & Zhang et al., 2018a \\
\hline Farnesene & $\begin{array}{l}\text { Increase the availability of acetyl-CoA by removing the native source of cytosolic acetyl-CoA } \\
(\Delta R H R 2) \text { and overexpressing xPK, PTA, ADA and NADH-HMGr }\end{array}$ & Fed-batch fermentation & $2.24 \mathrm{~g} / \mathrm{L} / \mathrm{h}>130 \mathrm{~g} / \mathrm{L}$ & Meadows et al., 2016 \\
\hline Oxygenated taxanes & $\begin{array}{l}\text { E. coli-S. cerevisiae co-culture by dividing the synthetic pathway for the acetylated diol paclitaxel } \\
\text { precursor into two modules }\end{array}$ & Co-culture in bioreactor & $33 \mathrm{mg} / \mathrm{L}$ & Zhou et al., 2015 \\
\hline Nerolidol & $\begin{array}{l}\text { Minimizing competition for FPP by destabilizing squalene synthase, degrade ER } \\
\text { membrane-integrating protein. }\end{array}$ & Two-phase flask & $4-5.5 \mathrm{~g} / \mathrm{L}$ & Peng et al., 2017 \\
\hline Casbene & Regulation of ERG2O and ERG9 by $\mathrm{P}_{\mathrm{HXT} 1}$ and $\mathrm{P}_{\text {ERG1 } 1}$ promoters & Deepwell microplate & $108.5 \mathrm{mg} / \mathrm{L}$ & Callari et al., 2018 \\
\hline Jolkinol C & Optimize soluble expression of Cbsp using protein tagging strategies, codon-optimization of CYPs & Milliliter plates & $800 \mathrm{mg} / \mathrm{L}$ & Wong et al., 2018 \\
\hline Carotenoid & $\begin{array}{l}\text { Colorimetric-based promoter strength comparison system; inducer/repressor-free sequential } \\
\text { control strategy by combining a modified GAL regulation system and a } \mathrm{P}_{\mathrm{HXT} 1 \text {-controlled squalene }} \\
\text { synthetic pathway }\end{array}$ & Fed-batch fermentation & $1156 \mathrm{mg} / \mathrm{L}$ & Xie et al., 2015 \\
\hline \multirow[t]{2}{*}{ Lycopene } & Lipid engineering; Improve triacylglycerol metabolism & Fed-batch fermentation & $2.37 \mathrm{~g} / \mathrm{L}$ & Ma et al., 2019 \\
\hline & Scaffold-free enzyme assemblies (IDI and CrtE); & Fed-batch fermentation & $2.3 \mathrm{~g} / \mathrm{L}$ & Kang W. et al., 2019 \\
\hline Medicagenic acid & $\begin{array}{l}\text { Endoplasmic reticulum (ER) engineering; expand the ER by disrupting the phosphatidic acid } \\
\text { phosphatase }\end{array}$ & Tube cultures & $27.1 \mathrm{mg} / \mathrm{L} 6$-fold & Arendt et al., 2017 \\
\hline$\beta$-Carotene & $\begin{array}{l}\text { Tri-functional CRISPR system combines transcriptional activation, transcriptional interference, and } \\
\text { gene deletion }\end{array}$ & Tube cultures & 2.8 -fold & Lian et al., 2017 \\
\hline Squalene & ER engineering; expand the ER by overexpressing a key ER size regulatory factor, INO2. & Shake flasks & $634 \mathrm{mg} / \mathrm{L}$ & Kim J.E. et al., 2019 \\
\hline \multicolumn{5}{|l|}{ E. coli } \\
\hline Total monoterpenoids & Non-natural route to isoprenoid biosynthesis (isoprenoid alcohol pathway/IPA) & Shake flasks & $0.6 \mathrm{~g} / \mathrm{L}$ & Clomburg et al., 2019 \\
\hline \multirow[t]{2}{*}{ Pinene } & $\begin{array}{l}\text { Adaptive laboratory evolution for improving pinene tolerance; E. coli co-culture system; whole-cell } \\
\text { biocatalysis }\end{array}$ & Shake flasks & $166.5 \mathrm{mg} / \mathrm{L}$ & Niu et al., 2018 \\
\hline & Cell-free enzyme systems for production of monoterpenes from glucose & Glass vials & $14.9 \mathrm{~g} / \mathrm{L}$ & Korman et al., 2017 \\
\hline Limonene & Cell-free enzyme systems & Glass vials & $12.5 \mathrm{~g} / \mathrm{L}$ & Korman et al., 2017 \\
\hline
\end{tabular}




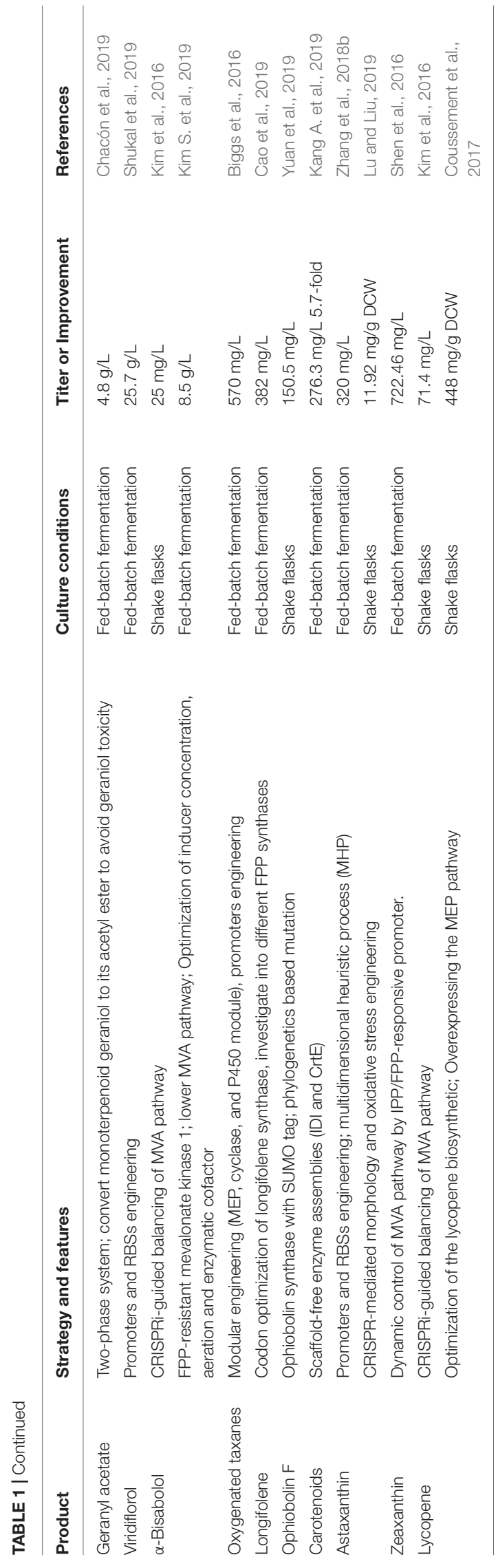

Protein tagging strategies are effective means for enzyme engineering. A truncated gene encoding casebene synthase from Jatropha curcas with various protein tags was integrated into a geranylgeranyl diphosphate (GGPP)-producing strain, which yields $160 \mathrm{mg} / \mathrm{L}$ of casbene (Wong et al., 2018). Using a small ubiquitin-like modifier (SUMO) fusion tag and phylogenetics based mutations, ophiobolin synthase solubility and activity were improved. The yield of sesterterpene ophiobolin F was increased to $150.51 \mathrm{mg} / \mathrm{L}$ in E. coli (Yuan et al., 2019). Endoplasmic reticulum-associated protein degradation decreased cellular levels of ERG9, and increased the titer of sesquiterpene nerolidol to $100 \mathrm{mg} / \mathrm{L}$ (Peng et al., 2017). Also, $\mathrm{N}$-degron-mediated destabilization of ERG20 improved the production of monoterpenes of $18 \mathrm{mg} / \mathrm{L}$ linalool or $76 \mathrm{mg} / \mathrm{L}$ limonene in S. cerevisiae (Peng et al., 2018). To decrease the concentration of pivotal enzyme, a synthetic degradation has been established based on Mesoplasma florum tmRNA system (Cameron and Collins, 2014). Based on the CRISPRi and the N-end rule for protein stability, Martínez et al. (2017) described a genome editing approach by changing the rates of both RNA synthesis and protein degradation. Synthetic protein scaffolds provide precise control of metabolic flux by preventing the loss of intermediates to diffusion or competing pathways (Dueber et al., 2009). However, scaffolded enzyme assemblies have different limitations, as enzymes fused in large structures may encounter a decrease or complete loss of the activity (Jia et al., 2014). Recently, Kang W. et al. (2019) developed a scaffold-free modular enzyme assembly by employing a pair of short peptide tags. The GGPP synthase and IDI were modularly assembled, which increased carotenoid production by 5.7 -folds in E. coli and yielded a titer of $2.3 \mathrm{~g} / \mathrm{L}$ lycopene in S. cerevisiae.

\section{REPROGRAMING AND DESIGN NEW PRECURSOR BIOSYNTHETIC PATHWAYS}

High intracellular levels of the essential intermediate IPP, may cause growth inhibition, reduce cell viability and plasmid instability (Martin et al., 2003; George et al., 2014, 2018). To explore more efficient and practical terpenoids biosynthetic pathways, non-natural pathways were developed. Kang et al. reported an alternative IPP-bypass MVA pathway by utilizing promiscuous activities of phosphomevalonate decarboxylase and an E. coli endogenous phosphatase, which successfully decoupled isopentenol production from IPP generation, and remarkably improved isoprenol titer to $3.7 \mathrm{~g} / \mathrm{L}$ in batch cultures (Kang et al., 2016; Kang A. et al., 2019). Clomburg et al. (2019) constructed an isoprenoid alcohol pathway (IPA) for terpenoids synthesis, which could convert isoprenoid precursors through a minimal number of steps, and less ATP consumption. Chatzivasileiou et al. (2019) established an isopentenol utilization pathway (IUP) for bioconversion of isopentenols, isoprenol, or prenol to IPP or DMAPP. The IUP is composed of choline kinase (from S. cerevisiae), isopentenyl phosphate kinase, and isopentenylpyrophosphate delta isomerase and requires ATP as its sole 
co-factor, whereas much more straightforward than the current MVA or MEP alternatives.

Acetyl-CoA is also the critical branch-point precursor for terpenoids biosynthesis. However, in S. cerevisiae, acetyl-CoA is compartmentalized that mainly derived from pyruvate in mitochondria and fatty acids degradation in the peroxisome (Hammer and Avalos, 2017). Meadows et al. (2016) rewired the central carbon metabolism of $S$. cerevisiae to improve redox balance and enable biosynthesis of cytosolic acetyl-CoA with a reduced ATP requirement. The engineered strains produced 25\% more farnesene while requiring $75 \%$ less oxygen, and sustaining stable yield for 2 weeks that reaches $>130 \mathrm{~g} / \mathrm{L}$ farnesene. This system has provided a reference for all terpenoids and other acetyl-CoA-derived compounds. Lu et al. (2019) constructed a synthetic acetyl-CoA pathway, in which, the catalytic activity of glycolaldehyde synthase was improved by directed evolution. Then the acetyl-phosphate synthase was selected based on the phylogenetic tree of PKs, which converts glycolaldehyde into acetyl-phosphate (AcP). AcP could be used to generate acetylCoA by the phosphate acetyltransferase. It is the shortest pathway from formaldehyde to acetyl-CoA.

In $S$. cerevisiae, NADPH production highly depends on the oxidative pentose phosphate pathway (Minard and McAlister-Henn, 2005). Kwak et al. (2019) engineered mutated phosphofructokinase (PFK) along with overexpression of glucose-6-phosphate dehydrogenase to reduce glycolytic metabolic fluxes, resulted in substantial increases of $[\mathrm{NADPH}] /\left[\mathrm{NADP}^{+}\right]$ratios. Moreover, amorpha-4,11-diene was overproduced in S. cerevisiae achieved a titer of $497 \mathrm{mg} / \mathrm{L}$ with a 3.7-fold increase compared to the parental strain.

\section{SUBCELLULAR ENGINEERING AND CELL FREE SYSTEM}

Compared with cytosol, mitochondria provide a compartmentalized environment with higher reducing redox potential. There is a growing interest in utilizing the acetylCoA pool in mitochondria for the biosynthesis of value-added compounds. By transplanting the whole FPP pathway together with amorpha-4,11-diene synthase into yeast mitochondria, the yield of amorpha-4,11-diene in engineered strain reached $427 \mathrm{mg} / \mathrm{L}$ (Yuan and Ching, 2016). Yee et al. (2019) targeted the geraniol biosynthetic pathway to the S. cerevisiae mitochondria to protect the GPP pool from consumption by the cytosolic ergosterol pathway. The production of geraniol in mitochondrial was 6-fold increase compared to cytosolic producing strains (Figure 1A). Lipid droplets (LDs) are ubiquitous organelles that store metabolic energy in the form of neutral lipids. Ma et al. (2019) established a lipophilic lycopene production strategy in S. cerevisiae by using LDs accumulation. A non-oleaginous S. cerevisiae for triacylglycerols production was combined with their composition adjustment and LDs size regulation. Therefore, lycopene accumulated continuously to $2.37 \mathrm{~g} / \mathrm{L}$ in 5 days (Figure 1B). Expansion of the endoplasmic reticulum (ER) could increase yeast metabolic capacity. Arendt et al. (2017) reported that the disruption of the phosphatidic acid phosphatase (PAH) resulted in the expansion of the ER, which stimulated the production of triterpene biosynthesis enzymes and increased triterpenoid and triterpene saponin accumulation. Kim J.E. et al. (2019) engineered S. cerevisiae to expand the ER by overexpressing a key ER size regulatory factor, INO2. The production of squalene was increased by 71 -fold, with the titer of $634 \mathrm{mg} / \mathrm{L}$ in shake flask fermentation (Figure 1C). Liu et al. (2020) compartmentalized yeast peroxisome as a subcellular factory for squalene biosynthesis. Hybridization of the cytoplasm- and peroxisome-engineered strains was constructed, and squalene with a titer of $11.0 \mathrm{~g} / \mathrm{L}$ was reached in two-stage fed-batch fermentation (Figure 1D).

On the other hand, cell free biosynthesis (CFB) systems are easy to use multiple enzyme pathways sourced from various organisms, and also overcome the challenges of precursor supply and products toxicity. The purified enzyme system and crude cell extract system are common application forms of CFB systems (Dudley et al., 2015; Li et al., 2018; Figure 1E). CFB systems designed by Korman et al. (2017) converted glucose into monoterpenes and can be self-sustaining for long periods. The platform contains 27 enzymes and using glycolysis reconstituted to generates both ATP, NADPH, and acetyl-CoA, resulting in the production of $12.5 \mathrm{~g} / \mathrm{L}$ limonene and $14.9 \mathrm{~g} / \mathrm{L}$ pinene. In addition, CFB systems provide great flexibility for biochemical pathways study. Chen et al. (2017) utilized in vitro metabolic engineering to reveal the regulatory network of a reconstituted amorpha-4,11diene synthetic pathway, and identified the inhibition of ATP on both FPP synthase and amorpha-4,11-diene synthase.

\section{CYTOCHROMES P450 AND POST-MODIFICATIONS OF TERPENOIDS}

Cytochromes P450 (P450s) play a crucial role in yielding final terpenoid products with wide chemical diversity and bioactivities. S. cerevisiae is a favored host for expressing P450s on account of advanced protein expression mechanism, abundant intracellular membranes and the inherent benefits of large-scale microbial fermentation (Paddon et al., 2013; Gold et al., 2018; Wong et al., 2018; Zhang et al., 2018a). It is generally deemed that the ability to express soluble P450s in E. coli is limited. The main challenge is the lack of an endomembrane system for attachment of the eukaryotic P450s, as they have a helical hydrophobic transmembrane domain containing 20-30 amino acid residues at their $\mathrm{N}$-terminal ends. Transmembrane domain truncation and $\mathrm{N}$-terminal replacement are vastly used for heterologous expression of eukaryotic P450s. Ichinose and Wariishi (2013) performed extensive heterologous expression of fungal P450s in E. coli using 304 of $\mathrm{P} 450$ isoforms and identified N-terminal amino acid sequences that can significantly improve chimeric P450s expression levels. They revealed that the choice of combinations of N-terminal and catalytic domains is critical for high-level expression. Biggs et al. (2016) demonstrated E. coli could be a feasible host for P450-mediated terpenoid biosynthesis. In their study, the first module, "MEP" was comprised of the rate-limiting enzymes of the IPP-producing MEP pathway. The second "cyclase" module was comprised of 


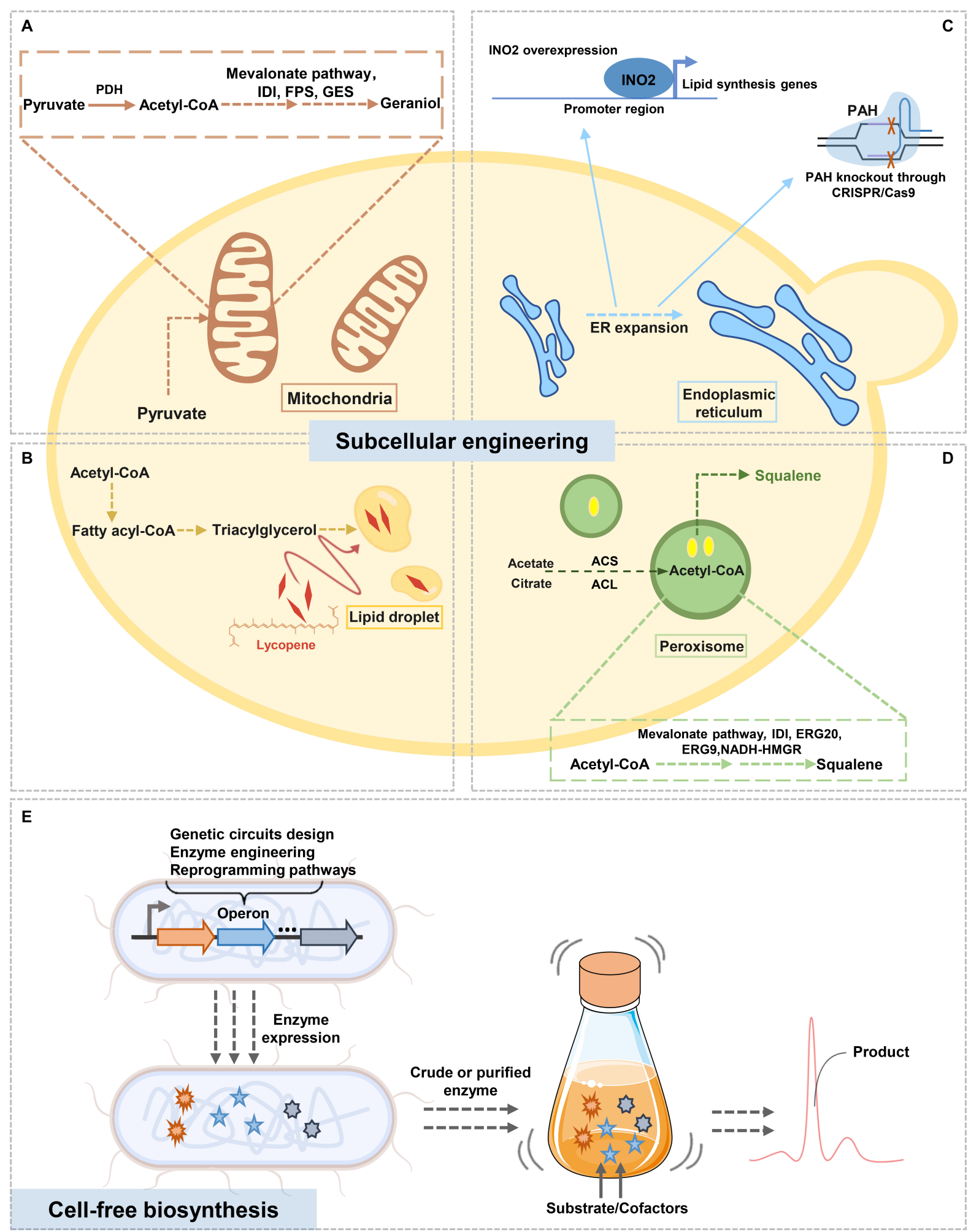

FIGURE 1 | Overview of terpenoids biosynthesis by subcellular engineering (A-D), and cell-free terpenoids biosynthesis (E). Yeast cells contain various subcellular organelles (e.g., mitochondria, endoplasmic reticulum, etc.) which provide beneficial circumstances for different terpenoids biosynthetic pathways. Cell-free biosynthesis systems by in vitro reconstructing the entire biosynthetic pathway is another efficient solution for terpenoid production. PDH, pyruvate dehydrogenase; IDI, isopentenyl diphosphate isomerase; FPS, farnesyl diphosphate synthase; GES, geraniol synthase; PAH, phosphatidic acid phosphatase; ACS, acetyl-CoA synthetase; ACL, ATP-dependent citrate lyase; ERG20, farnesyl diphosphate synthase; ERG9, squalene synthase; NADH-HMGR, NADH-specific HMG-CoA reductase. 
taxadiene synthase (TxS) and GGPP synthase. The relatively low expression of a five-copy plasmid with a weak promoter was essential for P450 and CPR functionality. Besides, with reductase partner interactions and $\mathrm{N}$-terminal modifications, a record yield of $570 \mathrm{mg} / \mathrm{L}$ of oxygenated taxanes was achieved in E. coli.

The important post-modifications of terpenoids also include hydroxylation by $\mathrm{P} 450$ s and glycosylation by glycosyltransferases. Gold et al. (2018) showed a platform for the production of steviol glucosides (SGs) in S. cerevisiae. Two P450s of kaurene oxidase $(\mathrm{KO})$ and kaurenoic acid hydroxylase (KAH) are required in succession in the conversion of kaurene into steviol. By optimizing the copy number modulation of $\mathrm{KO}-\mathrm{KAH}-$ CPR combinations, the conversion was maximized. Wang et al. (2015, 2019) established a series of cell factories to produce ginsenoside Rh2 by optimizing UDP-glycosyltransferase bioparts expression. Combined with precursor (protopanaxadiol) supply optimization, the titer of ginsenoside $\mathrm{Rh} 2$ reached $2.25 \mathrm{~g} / \mathrm{L}$ in fed-batch fermentation.

\section{CO-CULTURE OF ENGINEERED STRAINS}

A newly approach of co-culture engineering to enhance terpenoids production was developed. In some conditions, a single host cell cannot provide an optimal environment for functioning all pathway enzymes, and metabolic burdens from overexpression of complex pathways may reduce biosynthetic efficiency (Zhang et al., 2015; Wang et al., 2020). By dividing the acetylated diol paclitaxel precursor synthetic route into two modules, expressed in either S. cerevisiae or E. coli, a stable co-culture was achieved in the bioreactor. The engineered E. coli strain accomplishes the biosynthesis of the intermediate taxadiene. Meanwhile, S. cerevisiae is the preferred host for cytochrome P450 (P450s) expression, using this two-component system, oxygenated taxanes with a titer of $33 \mathrm{mg} / \mathrm{L}$ was overproduced (Zhou et al., 2015; Supplementary Figure 1D). Similarly, Niu et al. (2018) constructed an E. coli - E. coli co-culture system for pinene biosynthesis. The MEV pathway and heterologous pathway (the GPP synthase and pinene synthase) were engineered in different pinene tolerance E. coli strains, respectively. The optimization of whole-cell biocatalysis, which could separate cell growth and production phase, improved pinene production to $166.5 \mathrm{mg} / \mathrm{L}$.

\section{REFERENCES}

Ajikumar, P. K., Xiao, W. H., Tyo, K. E. J., Wang, Y., Simeon, F., Leonard, E., et al. (2010). Isoprenoid pathway optimization for Taxol precursor overproduction in Escherichia coli. Science 330, 70-74. doi: 10.1126/science.119 1652

Arendt, P., Miettinen, K., Pollier, J., de Rycke, R., Callewaert, N., and Goossens, A. (2017). An endoplasmic reticulum-engineered yeast platform for overproduction of triterpenoids. Metab. Eng. 40, 165-175. doi: 10.1016/j. ymben.2017.02.007

Bian, G., Deng, Z., and Liu, T. (2017). Strategies for terpenoid overproduction and new terpenoid discovery. Curr. Opin. Biotechnol. 48, 234-241. doi: 10.1016/j. copbio.2017.07.002

\section{CONCLUSION}

Over the last few decades, biological engineers achieved grand developments in synthetic biology. The enormous potential of $E$. coli and $S$. cerevisiae as platform strains has been confirmed with various successes. However, as synthetic biology targets are progressively more complicated, there remain some challenges to engineering industrial hosts because of the lack of knowledge of complex biochemical and cellular metabolism and its regulation. With the fast development of synthetic biology tools such as CRISPR-Cas9, adaptive laboratory evolution (ALE) combine with next-generation sequencing and high-throughput screening, it promises to reach a deeper understanding of cellular metabolism. The capacity of DNA synthesis has been made great progressed over the past decade, and it is conventional to synthesize the large gene cluster for terpenoids biosynthesis. In addition, new DNA assembly methods facilitate the speedy construction of different genetic part combinations or to replace genetic parts in a single step. Besides, dynamic control, compartmentalization, module design, or cell-free system are practical methods to enhance the overall reaction efficiency of multi-enzyme pathways.

\section{AUTHOR CONTRIBUTIONS}

Both authors conceived the review, wrote and reviewed the manuscript.

\section{FUNDING}

This research work was financially supported by the National Key R\&D Program of China (No. 2018YFC0311001), National Natural Science Foundation of China (Grant No. 81741153), and the Fundamental Research Funds for the Central Universities granted to $\mathrm{KH}$.

\section{SUPPLEMENTARY MATERIAL}

The Supplementary Material for this article can be found online at: https://www.frontiersin.org/articles/10.3389/fbioe. 2020.00347/full\#supplementary-material

Biggs, B. W., Lim, C. G., Sagliani, K., Shankar, S., Stephanopoulos, G., de Mey, M., et al. (2016). Overcoming heterologous protein interdependency to optimize P450-mediated Taxol precursor synthesis in Escherichia coli. Proc. Natl. Acad. Sci. U.S.A. 113, 3209-3214. doi: 10.1073/pnas.1515826113

Callari, R., Meier, Y., Ravasio, D., and Heider, H. (2018). Dynamic control of ERG20 and ERG9 expression for improved casbene production in Saccharomyces cerevisiae. Front. Bioeng. Biotechnol. 6:160. doi: 10.3389/fbioe. 2018.00160

Cameron, D. E., and Collins, J. J. (2014). Tunable protein degradation in bacteria. Nat. Biotechnol. 32, 1276-1281. doi: 10.1038/nbt.3053

Cao, Y., Zhang, R., Liu, W., Zhao, G., Niu, W., Guo, J., et al. (2019). Manipulation of the precursor supply for high-level production of longifolene by metabolically engineered Escherichia coli. Sci. Rep. 9:95. doi: 10.1038/s41598-018-36495-w 
Chacón, M. G., Marriott, A., Kendrick, E. G., Styles, M. Q., and Leak, D. J. (2019). Esterification of geraniol as a strategy for increasing product titre and specificity in engineered Escherichia coli. Microb. Cell Fact. 18:105. doi: 10.1186/s12934019-1130-0

Chatzivasileiou, A. O., Ward, V., Edgar, S. M., and Stephanopoulos, G. (2019). Two-step pathway for isoprenoid synthesis. Proc. Natl. Acad. Sci. U.S.A. 116, 506-511. doi: 10.1073/pnas.1812935116

Chen, H., Zhu, C., Zhu, M., Xiong, J., Ma, H., Zhuo, M., et al. (2019). High production of valencene in Saccharomyces cerevisiae through metabolic engineering. Microb. Cell Fact. 1:195. doi: 10.1186/s12934-019-1246-2

Chen, X., Zhang, C., Zou, R., Stephanopoulos, G., and Too, H. P. (2017). In vitro metabolic engineering of amorpha-4,11-diene biosynthesis at enhanced rate and specific yield of production. ACS Synth. Biol. 6, 1691-1700. doi: 10.1021/ acssynbio.6b0037

Cheng, S., Liu, X., Jiang, G., Wu, J., Zhang, J., Lei, D., et al. (2019). Orthogonal engineering of biosynthetic pathway for efficient production of limonene in Saccharomyces cerevisiae. ACS Synth. Biol. 8, 968-975. doi: 10.1021/acssynbio. $9 \mathrm{~b} 00135$

Clomburg, J. M., Crumbley, A. M., and Gonzalez, R. (2017). Industrial biomanufacturing: the future of chemical production. Science 355:aag0804. doi: 10.1126/science.aag0804

Clomburg, J. M., Qian, S., Tan, Z., Cheong, S., and Gonzalez, R. (2019). The isoprenoid alcohol pathway, a synthetic route for isoprenoid biosynthesis. Proc. Natl. Acad. Sci. U.S.A. 116, 12810-12815. doi: 10.1073/pnas.1821004116

Coussement, P., Bauwens, D., Maertens, J., and de Mey, M. (2017). Direct combinatorial pathway optimization. ACS Synth. Biol. 6, 224-232. doi: 10.1021/ acssynbio.6b00122

Dahl, R. H., Zhang, F., Alonso-Gutierrez, J., Baidoo, E., Batth, T. S., ReddingJohanson, A. M., et al. (2013). Engineering dynamic pathway regulation using stress-response promoters. Nat. Biotechnol. 31, 1039-1046. doi: 10.1038/nbt. 2689

Dudley, Q. M., Karim, A. S., and Jewett, M. C. (2015). Cell-free metabolic engineering: biomanufacturing beyond the cell. Biotechnol. J. 10, 69-82. doi: 10.1002/biot.201400330

Dueber, J. E., Wu, G. C., Malmirchegini, G. R., Moon, T. S., Petzold, C. J., Ullal, A. V., et al. (2009). Synthetic protein scaffolds provide modular control over metabolic flux. Nat. Biotechnol. 27, 753-759. doi: 10.1038/nbt.1557

Eriksen, D. T., Lian, J., and Zhao, H. (2014). Protein design for pathway engineering. J. Struct. Biol. 185, 234-242. doi: 10.1016/j.jsb.2013.03.011

George, K. W., Chen, A., Jain, A., Batth, T. S., Baidoo, E. E. K., Wang, G., et al. (2014). Correlation analysis of targeted proteins and metabolites to assess and engineer microbial isopentenol production. Biotechnol. Bioeng. 111, 1648-1658. doi: $10.1002 /$ bit. 25226

George, K. W., Thompson, M. G., Kim, J., Baidoo, E. E. K., Wang, G., Benites, V. T., et al. (2018). Integrated analysis of isopentenyl pyrophosphate (IPP) toxicity in isoprenoid-producing Escherichia coli. Metab. Eng. 47, 60-72. doi: 10.1016/j.ymben.2018.03.004

Gold, N. D., Fossati, E., Cetti Hansen, C., DiFalco, M., Douchin, V., and Martin, V. J. J. (2018). A Combinatorial Approach to study cytochrome P450 enzymes for de novo production of steviol glucosides in baker's yeast. ACS Synth. Biol. 7, 2918-2929. doi: 10.1021/acssynbio.8b00470

Hammer, S. K., and Avalos, J. L. (2017). Harnessing yeast organelles for metabolic engineering. Nat. Chem. Biol. 13, 823-832. doi: 10.1038/nchembio.2429

Hong, J., Park, S. H., Kim, S., Kim, S. W., and Hahn, J. S. (2019). Efficient production of lycopene in Saccharomyces cerevisiae by enzyme engineering and increasing membrane flexibility and NAPDH production. Appl. Microbiol. Biotechnol. 103, 211-223. doi: 10.1007/s00253-018-9449-8

Ichinose, H., and Wariishi, H. (2013). High-level heterologous expression of fungal cytochrome P450s in Escherichia coli. Biochem. Biophys. Res. Commun. 438, 289-294. doi: 10.1016/j.bbrc.2013.07.057

Jia, F., Narasimhan, B., and Mallapragada, S. (2014). Materials-based strategies for multi-enzyme immobilization and co-localization: a review. Biotechnol. Bioeng. 111, 209-222. doi: 10.1002/bit.25136

Jiang, G.-Z., Yao, M.-D., Wang, Y., Zhou, L., Song, T.-Q., Liu, H., et al. (2017). Manipulation of GES and ERG20 for geraniol overproduction in Saccharomyces cerevisiae. Metab. Eng. 41, 57-66. doi: 10.1016/j.ymben.2017.03.005
Kang, A., George, K. W., Wang, G., Baidoo, E., Keasling, J. D., and Lee, T. S. (2016). Isopentenyl diphosphate (IPP)-bypass mevalonate pathways for isopentenol production. Metab. Eng. 34, 25-35. doi: 10.1016/j.ymben.2015.12.002

Kang, A., Mendez-Perez, D., Goh, E.-B., Baidoo, E. E. K., Benites, V. T., Beller, H. R., et al. (2019). Optimization of the IPP-bypass mevalonate pathway and fed-batch fermentation for the production of isoprenol in Escherichia coli. Metab. Eng. 56, 85-96. doi: 10.1016/j.ymben.2019.09.003

Kang, W., Ma, T., Liu, M., Qu, J., Liu, Z., Zhang, H., et al. (2019). Modular enzyme assembly for enhanced cascade biocatalysis and metabolic flux. Nat. Commun. 10:4248. doi: 10.1038/s41467-019-12247-w

Kim, J. E., Jang, I. S., Son, S. H., Ko, Y. J., Cho, B. K., Kim, S. C., et al. (2019). Tailoring the Saccharomyces cerevisiae endoplasmic reticulum for functional assembly of terpene synthesis pathway. Metab. Eng. 56, 50-59. doi: 10.1016/j. ymben.2019.08.013

Kim, S., Kim, S. K., Seong, W., Woo, S., Lee, H., Yeom, S., et al. (2019). Enhanced (-)- $\alpha$-bisabolol productivity by efficient conversion of mevalonate in Escherichia coli. Catalysts 9:432. doi: 10.3390/catal9050432

Kim, S. K., Han, G. H., Seong, W., Kim, H., Kim, S.-W., Lee, D.-H., et al. (2016). CRISPR interference-guided balancing of a biosynthetic mevalonate pathway increases terpenoid production. Metab. Eng. 38, 228-240. doi: 10.1016/j.ymben. 2016.08.006

Korman, T. P., Opgenorth, P. H., and Bowie, J. U. (2017). A synthetic biochemistry platform for cell free production of monoterpenes from glucose. Nat. Commun. 8:15526. doi: 10.1038/ncomms15526

Kwak, S., Yun, E. J., Lane, S., Oh, E. J., Kim, K. H., and Jin, Y. (2019). Redirection of the glycolytic flux enhances isoprenoid production in Saccharomyces cerevisiae. Biotechnol. J. 15:1900173. doi: 10.1002/biot.201900173

Li, J., Zhang, L., and Liu, W. (2018). Cell-free synthetic biology for in vitro biosynthesis of pharmaceutical natural products. Synth. Syst. Biotechnol. 3, 83-89. doi: 10.1016/j.synbio.2018.02.002

Lian, J., Hamedirad, M., Hu, S., and Zhao, H. (2017). Combinatorial metabolic engineering using an orthogonal tri-functional CRISPR system. Nat. Commun. 8:1688. doi: 10.1038/s41467-017-01695-x

Liu, G.-S., Li, T., Zhou, W., Jiang, M., Tao, X.-Y., Liu, M., et al. (2020). The yeast peroxisome: a dynamic storage depot and subcellular factory for squalene overproduction. Metab. Eng. 57, 151-161. doi: 10.1016/j.ymben.2019.11.001

Lu, Q., and Liu, J. Z. (2019). Enhanced astaxanthin production in Escherichia coli via morphology and oxidative stress engineering. J. Agric. Food Chem. 67, 11703-11709. doi: 10.1021/acs.jafc.9b05404

Lu, X., Liu, Y., Yang, Y., Wang, S., Wang, Q., Wang, X., et al. (2019). Constructing a synthetic pathway for acetyl-coenzyme A from one-carbon through enzyme design. Nat. Commun. 10:1378. doi: 10.1038/s41467-019-09095-z

Ma, T., Shi, B., Ye, Z., Li, X., Liu, M., Chen, Y., et al. (2019). Lipid engineering combined with systematic metabolic engineering of Saccharomyces cerevisiae for high-yield production of lycopene. Metab. Eng. 52, 134-142. doi: 10.1016/j. ymben.2018.11.009

Martin, V. J. J., Piteral, D. J., Withers, S. T., Newman, J. D., and Keasling, J. D. (2003). Engineering a mevalonate pathway in Escherichia coli for production of terpenoids. Nat. Biotechnol. 21, 796-802. doi: 10.1038/nbt833

Martínez, V., Lauritsen, I., Hobel, T., Li, S., Nielsen, A. T., and Nørholm, M. H. H. (2017). CRISPR/Cas9-based genome editing for simultaneous interference with gene expression and protein stability. Nucleic Acids Res. 45, e171. doi: 10.1093/ nar/gkx797

Meadows, A. L., Hawkins, K. M., Tsegaye, Y., Antipov, E., Kim, Y., Raetz, L., et al. (2016). Rewriting yeast central carbon metabolism for industrial isoprenoid production. Nature 537, 694-697. doi: 10.1038/nature19769

Mendez-Perez, D., Alonso-Gutierrez, J., Hu, Q., Molinas, M., Baidoo, E. E. K., Wang, G., et al. (2017). Production of jet fuel precursor monoterpenoids from engineered Escherichia coli. Biotechnol. Bioeng. 114, 1703-1712. doi: 10.1002/ bit. 26296

Minard, K. I., and McAlister-Henn, L. (2005). Sources of NADPH in yeast vary with carbon source. J. Biol. Chem. 280, 39890-39896. doi: 10.1074/jbc.m509461200

Niu, F.-X., He, X., Wu, Y.-Q., and Liu, J.-Z. (2018). Enhancing production of pinene in Escherichia coli by using a combination of tolerance, evolution, and modular co-culture engineering. Front. Microbiol. 9:1623. doi: 10.3389/fmicb. 2018.01623 
Paddon, C. J., Westfall, P. J., Pitera, D. J., Benjamin, K., Fisher, K., McPhee, D., et al. (2013). High-level semi-synthetic production of the potent antimalarial artemisinin. Nature 496, 528-532. doi: 10.1038/nature12051

Peng, B., Nielsen, L. K., Kampranis, S. C., and Vickers, C. E. (2018). Engineered protein degradation of farnesyl pyrophosphate synthase is an effective regulatory mechanism to increase monoterpene production in Saccharomyces cerevisiae. Metab. Eng. 47, 83-93. doi: 10.1016/j.ymben.2018.02.005

Peng, B., Plan, M. R., Chrysanthopoulos, P., Hodson, M. P., Nielsen, L. K., and Vickers, C. E. (2017). A squalene synthase protein degradation method for improved sesquiterpene production in Saccharomyces cerevisiae. Metab. Eng. 39, 209-219. doi: 10.1016/j.ymben.2016.12.003

Pfleger, B. F., Pitera, D. J., Smolke, C. D., and Keasling, J. D. (2006). Combinatorial engineering of intergenic regions in operons tunes expression of multiple genes. Nat. Biotechnol. 24, 1027-1032. doi: 10.1038/nbt1226

Qi, L. S., Larson, M. H., Gilbert, L. A., Doudna, J. A., Weissman, J. S., Arkin, A. P., et al. (2013). Repurposing CRISPR as an RNA-guided platform for sequencespecific control of gene expression. Cell 152, 1173-1183. doi: 10.1016/j.cell.2013. 02.022

Scalcinati, G., Knuf, C., Partow, S., Chen, Y., Maury, J., Schalk, M., et al. (2012). Dynamic control of gene expression in Saccharomyces cerevisiae engineered for the production of plant sesquitepene $\alpha$-santalene in a fed-batch mode. Metab. Eng. 14, 91-103. doi: 10.1016/j.ymben.2012.01.007

Shen, H., Cheng, B., Zhang, Y., Tang, L., Li, Z., Bu, Y.-F., et al. (2016). Dynamic control of the mevalonate pathway expression for improved zeaxanthin production in Escherichia coli and comparative proteome analysis. Metab. Eng. 38, 180-190. doi: 10.1016/j.ymben.2016.07.012

Shukal, S., Chen, X., and Zhang, C. (2019). Systematic engineering for high-yield production of viridiflorol and amorphadiene in auxotrophic Escherichia coli. Metab. Eng. 55, 170-178. doi: 10.1016/j.ymben.2019.07.007

Wang, C., Liwei, M., Park, J. B., Jeong, S. H., Wei, G., Wang, Y., et al. (2018). Microbial platform for terpenoid production: Escherichia coli and Yeast. Front. Microbiol. 9:2460. doi: 10.3389/fmicb.2018.02460

Wang, P., Wei, W., Ye, W., Li, X., Zhao, W., Yang, C., et al. (2019). Synthesizing ginsenoside Rh2 in Saccharomyces cerevisiae cell factory at high-efficiency. Cell Discov. 5:5. doi: 10.1038/s41421-018-0075-5

Wang, P., Wei, Y., Fan, Y., Liu, Q., Wei, W., Yang, C., et al. (2015). Production of bioactive ginsenosides Rh2 and Rg3 by metabolically engineered yeasts. Metab. Eng. 29, 97-105. doi: 10.1016/j.ymben.2015.03.003

Wang, R., Zhao, S., Wang, Z., and Koffas, M. A. (2020). Recent advances in modular co-culture engineering for synthesis of natural products. Curr. Opin. Biotechnol. 62, 65-71. doi: 10.1016/j.copbio.2019.09.004

Westfall, P. J., Pitera, D. J., Lenihan, J. R., Eng, D., Woolard, F. X., Regentin, R., et al. (2012). Production of amorphadiene in yeast, and its conversion to dihydroartemisinic acid, precursor to the antimalarial agent artemisinin. Proc. Natl. Acad. Sci. U.S.A. 109, 111-118. doi: 10.1073/pnas.1110740109

Wong, A. S. T., Che, C.-M., and Leunga, K.-W. (2015). Recent advances in ginseng as cancer therapeutics: a functional and mechanistic overview. Nat. Prod. Rep. 32, 256-272. doi: 10.1039/c4np00080c

Wong, J., de Rond, T., d'Espaux, L., van der Horst, C., Dev, I., Rios-Solis, L., et al. (2018). High-titer production of lathyrane diterpenoids from sugar by engineered Saccharomyces cerevisiae. Metab. Eng. 45, 142-148. doi: 10.1016/j. ymben.2017.12.007

Xie, W., Ye, L., Lv, X., Xu, H., and Yu, H. (2015). Sequential control of biosynthetic pathways for balanced utilization of metabolic intermediates in Saccharomyces cerevisiae. Metab. Eng. 28, 8-18. doi: 10.1016/j.ymben.2014.11.007

Yee, D. A., DeNicola, A. B., Billingsley, J. M., Creso, J. G., Subrahmanyam, V., and Tang, Y. (2019). Engineered mitochondrial production of monoterpenes in Saccharomyces cerevisiae. Metab. Eng. 55, 76-84. doi: 10.1016/j.ymben.2019. 06.004

Yuan, J., and Ching, C. B. (2015). Dynamic control of ERG9 expression for improved amorpha-4,11-diene production in Saccharomyces cerevisiae. Microb. Cell Fact. 14:38. doi: 10.1186/s12934-015-0220-x

Yuan, J., and Ching, C.-B. (2016). Mitochondrial acetyl-CoA utilization pathway for terpenoid productions. Metab. Eng. 38, 303-309. doi: 10.1016/j.ymben.2016. 07.008

Yuan, W., Lv, S., Chen, L., Zhao, Y., Deng, Z., and Hong, K. (2019). ). Production of sesterterpene ophiobolin by a bifunctional terpene synthase in Escherichia coli. Appl. Microbiol. Biotechnol. 103, 8785-8797. doi: 10.1007/s00253-019-10 103-x

Zhang, C., Liu, J., Zhao, F., Lu, C., Zhao, G.-R., and Lu, W. (2018a). Production of sesquiterpenoid zerumbone from metabolic engineered Saccharomyces cerevisiae. Metab. Eng. 49, 28-35. doi: 10.1016/j.ymben.2018. 07.010

Zhang, C., Seow, V. Y., Chen, X., and Too, H. P. (2018b). Multidimensional heuristic process for high-yield production of astaxanthin and fragrance molecules in Escherichia coli. Nat. Commun. 9:1858. doi: 10.1038/s41467-01804211-X

Zhang, H., Pereira, B., Li, Z., Stephanopoulos, G., and Demain, A. L. (2015). Engineering Escherichia coli coculture systems for the production of biochemical products. Proc. Natl. Acad. Sci. U.S.A. 112, 8266-8271. doi: 10 1073/pnas.1506781112

Zhao, J., Li, C., Zhang, Y., Shen, Y., Hou, J., and Bao, X. (2017). Dynamic control of ERG20 expression combined with minimized endogenous downstream metabolism contributes to the improvement of geraniol production in Saccharomyces cerevisiae. Microb. Cell Fact. 16:17. doi: 10.1186/s12934-0170641-9

Zhou, K., Qiao, K., Edgar, S., and Stephanopoulos, G. (2015). Distributing a metabolic pathway among a microbial consortium enhances production of natural products. Nat. Biotechnol. 33, 377-383. doi: 10.1038/nbt.3095

Conflict of Interest: The authors declare that the research was conducted in the absence of any commercial or financial relationships that could be construed as a potential conflict of interest.

Copyright (c) 2020 Zhang and Hong. This is an open-access article distributed under the terms of the Creative Commons Attribution License (CC BY). The use, distribution or reproduction in other forums is permitted, provided the original author(s) and the copyright owner(s) are credited and that the original publication in this journal is cited, in accordance with accepted academic practice. No use, distribution or reproduction is permitted which does not comply with these terms. 